\title{
Posterolateral spinal fusion with ostegenesis induced BMSC seeded TCP/HA in a sheep model
}

\begin{abstract}
Autogenous bone graft is the gold standard for fusion procedure. However, pain at donor site and inconsistent outcome have left a surgeon to venture into some other technique for spinal fusion. The objective of this study was to determine whether osteogenesis induced bone marrow stem cells with the combination of ceramics granules (HA or TCP/HA), and fibrin could serve as an alternative to generate spinal fusion. The sheep's bone marrow mesenchymal stem cells (BMSCs) were aspirated form iliac crest and cultured for several passages until confluence. BMSCs were trypsinized and seeded on hydroxyapatite scaffold (HA) and tricalcium phosphate/hydroxyapatite (TCP/HA) for further osteogenic differentiation in the osteogenic medium one week before implantation. Six adult sheep underwent three-level, bilateral, posterolateral intertransverse process fusions at L1-L6. Three fusion sites in each animal were assigned to three treatments: (a) HA constructs group/L1-L2, (b) TCP/HA constructs group/L2-L3, and (c) autogenous bone graft group/L5L6. The spinal fusion segments were evaluated using radiography, manual palpation, histological analysis and scanning electron microscopy (SEM) 12 weeks post implantation. The TCP/HA constructs achieved superior lumbar intertransverse fusion compared to HA construct but autogenous bone graft still produced the best fusion among all.
\end{abstract}

Keyword: Bone tissue engineering; Hydroxyapatite; Tricalcium phosphate; Spinal fusion; Fibrin 